(4) Insbesondere für die Wahlentscheidung zugunsten der CDU und der SPD sind bundespolitische Einflüsse von größerer Relevanz als landespolitische Faktoren. Die Entscheidung für die Linkspartei/PDS wird hingegen eher von landespolitischen Faktoren bestimmt.

\title{
Landtagswahlen in Hessen 1991 bis 2003: bundespolitische Einflüsse vor allem bei unabhängigen Wählern
}

\author{
Tatjana Rudi
}

Hessisches Landtagswahlverhalten scheint maßgeblich von bundespolitischen Faktoren beeinflusst zu sein. So lautete schon 1954 der Wahlslogan der hessischen CDU: „Deine Wahl im Hessenstaat zählt im Bonner Bundesrat. Regierung Zinn stützt Ollenhauer, wählt CDU für Adenauer. "1 In der zur hessischen Landtagswahl 1982 in der ZParl publizierten Analyse wird festgestellt, dass bundespolitische Einflüsse „die Wahlabsichten der Hessen noch unmittelbar vor dem Wahltermin in bisher nicht beobachtetem Umfang “ korrigierten ${ }^{2}$, und bei der viel beachteten Landtagswahl 1999 war „der Unmut vieler Wähler über die Ausländerpolitik der neuen Bundesregierung, in dem sich auch die wachsende allgemeine Unzufriedenheit mit den Leistungen der neuen Mannschaft in Bonn aktualisieren konnte" ${ }^{\text {"3 }}$, entscheidend für das Wählervotum. Auch 2003 trug die „massive Unzufriedenheit mit der gerade wiedergewählten rot-grünen Bundesregierung “4 zum Wahlerfolg der CDU in Hessen bei. Folgt man der Literatur, so fungieren hessische Landtagswahlen folglich als „Stimmungstests für ... Bundesregierungen "5. Inwiefern diese weit verbreitete Position tatsächlich zutrifft, wurde jedoch vergleichsweise selten systematisch getestet. ${ }^{6}$ Dies soll im Folgenden mithilfe von Individualdatenanalysen für die hessischen Landtagswahlen von 1991 bis 2003 unternommen werden, die auch nicht-bundespolitische Erklärungsfaktoren berücksichtigen. ${ }^{7}$

1 Zitiert nach Gerhard Lehmbruch, Parteienwettbewerb im Bundesstaat, 2. Auflage, Opladen 1998, S. 139, Hervorhebung nicht im Original.

2 Gerhard Franz / Robert Danziger / Jürgen Wiegand, Die hessische Landtagswahl vom 26. September 1982: Unberechenbarkeit der Wählerpsyche oder neue Mehrheiten?, in: ZParl, 14. Jg. (1983), H. 1, S. 62.

3 Rüdiger Schmitt-Beck, Die hessische Landtagswahl vom 7. Februar 1999: Der Wechsel nach dem Wechsel, in: ZParl, 30. Jg. (2000), H. 1, S. 17.

4 Rüdiger Schmitt-Beck / Cornelia Weins, Die hessische Landtagswahl vom 2. Februar 2003: Erstmals Wiederwahl einer CDU-Regierung, in: ZParl, 34. Jg. (2003), H. 4, S. 671.

5 Rüdiger Schmitt-Beck, a.a.O. (2000), S. 4.

6 Siehe als Ausnahmen Rüdiger Schmitt-Beck / Cornelia Weins, a.a.O.; Daniela Klos, Motivtransfer bei Nebenwahlen: Ein Vergleich wahlspezifischer und bundespolitischer Einflussfaktoren auf die Wahlentscheidung bei der hessischen Landtagswahl und der Europawahl in Deutschland 1999, in: Frank Brettschneider / Jan van Deth / Edeltraud Roller (Hrsg.), Europäische Integration der öffentlichen Meinung, Opladen 2003, S. 335 - 359.

7 Datengrundlage bilden die im ZA archivierten Umfragedaten: ZA-Nr. 2116, 2582, 3120, 3866. 


\section{Erklärungsfaktoren bei hessischen Landtagswahlen im Überblick}

Zur Analyse des hessischen Landtagswahlverhaltens wird auf den sozialpsychologischen Ansatz mit seinen drei Faktoren Parteiidentifikation, Kandidaten- und Sachfragenorientierung zurückgegriffen. ${ }^{8}$ Während die als vergleichsweise stabil betrachtete Parteiidentifikation ebenenunspezifisch ist, können sich die beiden kurzfristig variablen Orientierungen zu den Kandidaten und Sachfragen sowohl auf die Bundes- als auch auf die Landesebene beziehen.

Bevor das Augenmerk den bundespolitischen Faktoren zugewendet wird, soll zunächst die Verteilung der stabilen ebenenunspezifischen Parteiidentifikation betrachtet werden, die das stabile Wählerpotential der Parteien bestimmt. Dabei sind vor allem zwei Entwicklungen hervorzuheben: Erstens ist zwischen 1991 und 2003 in Hessen der Anteil von Personen ohne Parteiidentifikation (beziehungsweise mit einer Identifikation zu einer Splitterpartei) leicht von 26 auf 33 Prozent angestiegen. Zweitens ist der Anteil der Personen, die der SPD zuneigen, von 33 auf 26 Prozent gesunken. Sofern diese Schwankungen nicht stichprobenbedingt sind, ist dies ein weiteres Zeichen für den (langsamen) Niedergang der „roten Bastion Hessen"9. Dagegen sind die Werte für die anderen Parteien vergleichsweise stabil, wobei sich seit 1995 nur noch zwei Prozent mit den Freidemokraten identifizieren. Folglich ist die FDP in besonderem Maße darauf angewiesen, Wähler über die beiden kurzfristigen Orientierungen für sich zu gewinnen.

Die Verteilung der bundespolitischen Faktoren in der hessischen Wählerschaft zeigt hinsichtlich der Bewertungen der Bundespolitiker, dass die Christdemokraten über die Zeit relativ ähnlich bewertet werden, während die Haltung zu den SPD-Spitzenpolitikern deutlicheren Schwankungen unterliegt. So genoss der schleswig-holsteinische Ministerpräsident und designierte SPD-Vorsitzende Björn Engholm 1991 einen leichten Sympathievorsprung vor dem amtierenden Bundeskanzler Helmut Kohl. Ähnlich sah es 1999 aus: Der frisch gekürte sozialdemokratische Bundeskanzler Gerhard Schröder wurde im Durchschnitt etwas besser bewertet als Wolfgang Schäuble. Wiesen die Spitzenpolitiker in den Jahren 1991 und 1999 noch vergleichsweise ähnliche Sympathiewerte auf, so war dies vor der hessischen Landtagswahl 2003 nicht der Fall. Hier lag die CDU-Vorsitzende Angela Merkel in der Beliebtheit bei den hessischen Wählern eindeutig vor dem 2002 gerade wieder gewählten Bundeskanzler Schröder.

Bei Betrachtung des zweiten bundespolitischen Faktors Zufriedenheit mit der Bundesregierung fällt auf, dass diese deutlich schwankt. Während die hessischen Wähler 1991 unmittelbar nach der ersten gesamtdeutschen Bundestagswahl, bei der die schwarz-gelbe Koalition einen klaren Sieg errungen hatte, noch vergleichsweise zufrieden waren, wurde diese 1995 deutlich schlechter bewertet. Ein ähnlicher Befund lässt sich auch für die rot-grüne Bundesregierung in den Jahren 1999 und 2003 konstatieren - jedoch auch auf einem etwas niedrigeren Ausgangsniveau.

8 Vgl. die Ausführungen von Oscar W. Gabriel und Everhard Holtmann in diesem Heft der ZParl; Herbert B. Asher, Voting Behavior Research in the 1980s: An Examination of Some Old and New Problem Areas, in: Ada W. Finifter (Hrsg.), Political Science: The State of the Discipline, Washington D.C. 1983, S. 339 - 388. Grundlegend zum sozialpsychologischen Ansatz: Angus Campbell I Philip E. Converse / Warren E. Miller / Donald E. Stokes, The American Voter, New York 1960.

9 Gerhard Franz / Robert Danziger / Jürgen Wiegand, a.a.O., S. 64. 


\begin{tabular}{|c|c|c|c|c|}
\hline \multicolumn{5}{|c|}{$\begin{aligned} \text { Tabelle 1: } & \text { Einstellungen zu den Bundespolitikern der SPD und CDU sowie zur Bundes- und } \\
& \text { Landesregierung in Hessen, } 1991 \text { bis } 2003 \text { (Mittelwerte auf einer Skala von } 1 \text { bis 11) }\end{aligned}$} \\
\hline & $\begin{array}{l}\text { SPD-Bundes- } \\
\text { politiker }\end{array}$ & $\begin{array}{l}\text { CDU-Bundes- } \\
\text { politiker }\end{array}$ & $\begin{array}{c}\text { Bundes- } \\
\text { regierung }\end{array}$ & $\begin{array}{c}\text { Landes- } \\
\text { regierung }\end{array}$ \\
\hline 1991 & 8,39 & 7,64 & 7,56 & 6,86 \\
\hline 1995 & - & - & 6,03 & 6,64 \\
\hline 1999 & 7,86 & 7,18 & 6,57 & 6,59 \\
\hline 2003 & 5,81 & 7,06 & 5,09 & 7,01 \\
\hline
\end{tabular}

Erheblich weniger verändert sich die Zufriedenheit mit der Landesregierung im Zeitverlauf. ${ }^{10}$ Interessanterweise wurde jedoch die schwarz-gelbe Bundesregierung 1991 besser bewertet als die hessische Landesregierung derselben politischen Couleur. Dagegen waren die Hessen 2003 mit der CDU-FDP-Landesregierung deutlich zufriedener als mit der rot-grünen Bundesregierung.

Bezüglich der Kandidatenpräferenz ist festzustellen, dass die hessischen Wähler 1991 knapp den amtierenden Ministerpräsidenten Walter Wallmann von der CDU vor dem SPD-Herausforderer Hans Eichel bevorzugten (45 zu 40 Prozent). Als Ministerpräsident war letzterer 1995 etwa genau so beliebt wie sein CDU-Gegner Manfred Kanther. Demgegenüber wies Eichel 1999 mit 47 Prozent einen deutlichen Vorsprung in der Wählergunst vor Roland Koch mit 30 Prozent auf, der ihm bekanntlich aber nichts nützte. 2003 ist eine ebenso eindeutige Präferenz für den Regierungschef Koch gegenüber seinem Kontrahenten Gerhard Bökel zu verzeichnen (48 zu 32 Prozent). Insgesamt genossen also die amtierenden Ministerpräsidenten mit Ausnahme des Jahres 1995 einen Popularitätsvorsprung.

2003 legte bereits die Kenntnis dieser Aggregatverteilungen das Wahlergebnis nahe, denn die CDU war praktisch auf allen Gebieten im Vorteil: Mehr Wähler bekundeten eine christdemokratische Parteiidentifikation als eine sozialdemokratische; die Zufriedenheit mit der Bundesregierung war relativ gering, mit der Landesregierung dagegen relativ hoch; $A n$ gela Merkel wurde im Vergleich zu Gerhard Schröder deutlich positiver bewertet; und es gab eine eindeutige Präferenz in der hessischen Bevölkerung zugunsten des Ministerpräsidenten Roland Koch. Damit ist aber noch nichts über die jeweilige Bedeutung dieser Faktoren für die Wahlentscheidung ausgesagt: Ob der deutliche Wahlsieg der CDU im Jahre 2003 auf die Parteiidentifikation, auf landes- oder bundespolitische Faktoren zurückzuführen ist, bleibt unbestimmt.

$10 \mathrm{Ob}$ diese Bewertung tatsächlich zutrifft oder nur ein methodisches Artefakt ist, weil der Mehrheit der Bürger möglicherweise nicht ausreichend Informationen zur Verfügung stehen, um die Landesregierung angemessen bewerten zu können, lässt sich mit den zur Verfügung stehenden Daten nicht beantworten. 


\section{Die Einschätzung bundespolitischer Faktoren durch die Wähler der hessischen Parteien}

Nachdem die Verteilung der einzelnen Variablen im Aggregat betrachtet wurde, soll im Folgenden zunächst beleuchtet werden, wie die Wählerschaft der hessischen Parteien bundespolitische Größen, die den Wahlausgang in den Ländern beeinflussen können, bewertet. Dabei wird wieder zwischen der Zufriedenheit mit den führenden Bundespolitikern (Bundeskanzler, Vorsitzender der größten Oppositionspartei) und den Leistungen der Bundesregierung unterschieden (vgl. Tabellen 2 und 3).

Die Einstellungen zu den führenden Bundespolitikern decken sich mit dem, was man plausiblerweise erwartet. CDU-Bundespolitiker erfreuen sich bei den Wählern der eigenen Partei und denen der FDP einer größeren Beliebtheit als Spitzenpolitiker der SPD, die Wähler der SPD und der Grünen geben den SPD-Politikern den Vorzug. Diese Konstellation zeigt sich bei allen drei untersuchten Wahlen, bei der FDP aber nicht so stark. Spitzenwerte in der Popularität im eigenen Lager erzielten Helmut Kohl (1991), Gerhard Schröder (1999) und Björn Engholm (1991). Beim potenziellen Koalitionspartner schneiden die Politiker der beiden großen Volksparteien stets etwas schlechter ab als in der Anhängerschaft der eigenen Partei.

\begin{tabular}{|l|l|c|c|c|c|c|}
\hline \multicolumn{2}{|c|}{ Tabelle 2: Bewertung von Bundespolitikern der SPD und der CDU in der Wählerschaft der } \\
hessischen Parteien, 1991 bis 2003 (Mittelwerte auf einer Skala von 1 bis 11)
\end{tabular}

Bei der Zufriedenheit mit der Bundesregierung lassen sich die gleichen Muster erkennen. In der Amtszeit der CDU/CSU/FDP-Bundesregierung (1991, 1995) waren die Wähler der Koalitionsparteien deutlich zufriedener mit der Bundesregierung als die Wähler von SPD

\begin{tabular}{|l|c|c|c|c|c|}
\hline Tabelle 3: Zufriedenheit mit der Leistung der Bundesregierung in der Wählerschaft der hessischen \\
Parteien, 1991 bis 2003 Mittelwerte auf einer Skala von 1 bis 11)
\end{tabular}


und Bündnis 90/Die Grünen, in der Zeit der rot-grünen Koalition (1999, 2003) verhielt es sich umgekehrt. Die Grünen-Wähler standen der Regierung Kohl stets kritischer gegenüber als die Anhänger der SPD, während sich CDU/CSU- und FDP-Wähler in ihren Einstellungen zur rot-grünen Bundesregierung kaum voneinander unterschieden.

\section{Bundespolitische Bestimmungsfaktoren der Wahlabsicht hessischer Wähler}

Mit dem Befund, dass die Wähler der hessischen Landtagsparteien bundespolitische Faktoren sehr unterschiedlich bewerten, ist noch nichts darüber ausgesagt, welche Rolle die Einstellungen zu führenden Bundespolitikern und zur Bundesregierung für die Erklärung der Wahlabsicht bei Landtagswahlen spielen. Zusätzlich zu dieser Veränderung der Analyseperspektive (Wahlabsicht als abhängige Variable) wird das Erklärungsmodell um zwei Faktoren erweitert, die das sozialpsychologische Modell als wichtige Bestimmungsgrößen des individuellen Wahlentscheids behandelt: die Parteiidentifikation und die Einstellung zu den Spitzenkandidaten bei der jeweiligen Landtagswahl. Das Ziel der Untersuchung besteht also darin nachzuweisen, ob die bundespolitischen Faktoren unter Kontrolle der anderen Erklärungsvariablen das Wahlverhalten im Land beeinflussen ${ }^{11}$. An Stelle der Absicht zur Wahl der vier im Hessischen Landtag vertretenen Parteien wurden SPD und Grüne zu einer „rot-grünen“, CDU und FDP zu einer „schwarz-gelben“ Allianz zusammengefasst. Dies entspricht der Struktur des Parteienwettbewerbs bei den untersuchten Wahlen, da die Wähler auf Grund expliziter oder impliziter Koalitionsaussagen stets vor der Alternative standen, sich für eines dieser beiden Bündnisse zu entscheiden ${ }^{12}$.

In den Tabellen 4 und 5 sind verschiedene Einstellungskonstellationen wiedergegeben, unter denen der Einfluss bundespolitischer Faktoren wirksam werden kann. Die erste Gruppe von Wählern besteht aus Personen ohne Parteiidentifikation, die keinen der beiden Kandidaten für das Amt des Ministerpräsidenten favorisieren. Die zweite Gruppe umfasst die Parteianhänger, die aber ebenfalls keine Kandidatenpräferenz erkennen lassen. Die Angehörigen der dritten Gruppe dagegen identifizieren sich mit einer der vier Parteien und favorisieren zudem den Spitzenkandidaten des politischen Lagers, dem sie sich zugehörig fühlen. Für jede dieser drei Gruppen erfolgt eine Schätzung des Einflusses der Bundespolitik. Dabei werden zwei bundespolitische Einstellungskonstellationen miteinander verglichen: In der ersten bewerten die Befragten die Bundespolitik (Leistung der Bundesregie-

11 Diesen Schätzmodellen liegen multinomiale logistische Regressionen zu Grunde. Es wurde ein sparsames und zwischen den Wahlen weitgehend vergleichbares Modell geschätzt, das die nachfolgend aufgeführten Variablen enthält: Dummyvariablen für die Parteiidentifikation zu den beiden Lagern, jeweils eine Variable zur Abbildung der Zufriedenheit mit der Bundesregierung beziehungsweise der Landesregierung, Dummyvariablen, die die Präferenz für einen Ministerpräsidentenkandidaten abbilden und ein bundespolitisches Kandidatendifferential. Für das Kandidatendifferential wurde der Sympathiewert des SPD-Bundespolitikers vom Sympathiewert des beziehungsweise der CDU-Bundespolitikerin subtrahiert. Positive Werte indizieren damit eine positivere Einschätzung des beziehungsweise der CDU-Bundespolitikerin gegenüber dem SPDBundespolitiker.

12 Vgl. Wilhelm Bürklin / Gerhard Franz / Rüdiger Schmitt, Die hessische Landtagswahl vom 25. September 1983: Politische Neuordnung nach der Wende?, in: ZParl, 15. Jg. (1984), H. 2, S. 243 - 249. Auch die bivariaten Ergebnisse zu den Zusammenhängen zwischen bundespolitischen Faktoren und der Wahlentscheidung legen dies nahe. 
rung in Tabelle 4; Spitzenpolitiker auf der Bundesebene in Tabelle 5) extrem negativ, in der zweiten liegt eine extrem positive Bewertung der Bundespolitik vor. Der Vergleich dieser beiden Werte gibt den bedingten Einfluss der Bundespolitik auf die Wahlwahrscheinlichkeit bei Landtagswahlen an ${ }^{13} .1991$ und 1995 müsste eine positive Bewertung der Bundesregierung die Wahrscheinlichkeit zur Wahl von CDU und FDP erhöht und die Präferenz für rot-grün abgeschwächt haben, in den Jahren 1998 und 2003 ist dagegen im Falle eines Rückenwindes aus Berlin eine steigende Wahrscheinlichkeit zur Wahl von Rot-Grün zu erwarten.

Diese Annahme bestätigt sich in allen untersuchten Konstellationen, zeigt sich aber am deutlichsten in der Gruppe der Unabhängigen. 1991 erklärten nur acht Prozent der Unabhängigen, die mit der Bundesregierung sehr unzufrieden waren, bei der Landtagswahl für die CDU oder die FDP votieren zu wollen. In der Gruppe der Zufriedenen lag der Anteil potenzieller CDU/FDP-Wähler bei 42 Prozent. In die entgegengesetzte Richtung wirkt der Einfluss der Bundespolitik im rot-grünen Lager: Starke Unzufriedenheit mit den Leistungen der Bundesregierung fördert die Neigung, die SPD oder die Grünen zu wählen (53 Prozent); bei den mit der Bundesregierung extrem zufriedenen Bürgern ist die Wahrscheinlichkeit hierfür nur halb so groß (28 Prozent). Die gleichen Wirkungen der Bundespolitik

\begin{tabular}{|c|c|c|c|c|c|}
\hline \multicolumn{6}{|c|}{$\begin{array}{l}\text { Tabelle 4: Wirkung der Einstellung zur Leistung der Bundesregierung auf die Wablentscheidung } \\
\text { verschiedener Wählertypen in Hessen, } 1991 \text { bis } 2003 \text { (Angaben in Prozent) }\end{array}$} \\
\hline & & \multicolumn{2}{|c|}{ Wahl SPD-Grüne } & \multicolumn{2}{|c|}{ Wahl CDU-FDP } \\
\hline & & \begin{tabular}{|r|} 
Unzufrieden- \\
heit mit BReg.
\end{tabular} & $\begin{array}{l}\text { Zufriedenheit } \\
\text { mit BReg. }\end{array}$ & $\begin{array}{r}\text { Unzufrieden- } \\
\text { heit mit BReg. }\end{array}$ & $\begin{array}{l}\text { Zufriedenheit } \\
\text { mit BReg. }\end{array}$ \\
\hline \multirow[t]{3}{*}{1991} & Unabhängige & 53 & 28 & 8 & 42 \\
\hline & Parteianhänger & 88 & 77 & 39 & 82 \\
\hline & $\begin{array}{l}\text { Parteianhänger mit gleicher } \\
\text { Ministerpräsidentenpräferenz }\end{array}$ & 98 & 96 & 66 & 94 \\
\hline \multirow[t]{3}{*}{1995} & Unabhängige & 63 & 15 & 1 & 71 \\
\hline & Parteianhänger & 93 & 69 & 17 & 97 \\
\hline & $\begin{array}{l}\text { Parteianhänger mit gleicher } \\
\text { Ministerpräsidentenpräferenz }\end{array}$ & 100 & 90 & 64 & 100 \\
\hline \multirow[t]{3}{*}{1999} & Unabhängige & 26 & 66 & 55 & 26 \\
\hline & Parteianhänger & 61 & 90 & 88 & 85 \\
\hline & $\begin{array}{l}\text { Parteianhänger mit gleicher } \\
\text { Ministerpräsidentenpräferenz }\end{array}$ & 79 & 96 & 90 & 83 \\
\hline \multirow[t]{3}{*}{2003} & Unabhängige & 21 & 71 & 64 & 21 \\
\hline & Parteianhänger & 64 & 94 & 77 & 54 \\
\hline & $\begin{array}{l}\text { Parteianhänger mit gleicher } \\
\text { Ministerpräsidentenpräferenz }\end{array}$ & 87 & 98 & 94 & 87 \\
\hline \multicolumn{6}{|c|}{$\begin{array}{l}\text { gleiche Ministerpräsidentenpräferenz = gleichgerichtete Ministerpräsidentenpräferenz; BReg. = Bun- } \\
\text { desregierung. } \\
\text { Eingetragen sind die geschätzten Wahlwahrscheinlichkeiten der Lager für vollkommene Zufriedenheit } \\
\text { und vollkommene Unzufriedenheit mit der Bundesregierung. } \\
\text { Quelle: Eigene Berechnungen. }\end{array}$} \\
\hline
\end{tabular}

13 Die übrigen Variablen werden, sofern sie nicht zur Charakterisierung der jeweiligen Wählertypen verwendet werden, auf dem Modus beziehungsweise dem arithmetischen Mittel konstant gehalten 
zeigen sich bei den Parteianhängern ohne Kandidatenpräferenz sowie bei den Parteiidentifizierern, die den Spitzenkandidaten ihres Lagers bevorzugen. Die für das Jahr 1995 durchgeführten Analysen lassen die gleiche Wirkungsrichtung erkennen. Nach dem Regierungswechsel 1998 profitierten, wie erwartet, SPD und Grüne von positiv bewerteten bundespolitischen Rahmenbedingungen, CDU und FDP wurden durch günstige bundespolitische Einflussfaktoren benachteiligt.

Die Einstellungen zu den führenden Bundespolitikern wirken sich ganz ähnlich auf die Wahlabsicht bei Landtagswahlen aus. In der Gruppe der Unabhängigen, die Björn Engholm (SPD) uneingeschränkt positiv, Helmut Kohl (CDU) aber uneingeschränkt negativ bewerteten, belief sich die Wahrscheinlichkeit einer Wahl von Rot-Grün auf 64 Prozent. Dagegen erklärten bei einer sehr positiven Bewertung des Kanzlers und einer sehr negativen Bewertung des Oppositionsführers nur 13 Prozent der Befragten, sich für SPD oder Bündnis 90/Die Grünen entscheiden zu wollen. Eine für Schwarz-Gelb günstige Bewertung der personellen Konstellation auf Bundesebene hatte den entgegengesetzten Effekt. Die Wahlwahrscheinlichkeit stieg beim Übergang von stark negativen zu stark positiven Einstellungen von 10 auf 51 Prozent. Auch hier zeigen sich 1991 und 1995 gleiche Muster: Der Regierungswechsel löste die gleichen Effekte aus wie die Zufriedenheit mit den Leistungen der Bundesregierung.

\begin{tabular}{|c|c|c|c|c|c|}
\hline \multicolumn{6}{|c|}{$\begin{array}{l}\text { Tabelle 5: Wirkung der Einstellung zu Bundespolitikern auf die Wablentscheidung verschiedener } \\
\text { Wählertypen in Hessen, } 1991 \text { bis } 2003 \text { (Angaben in Prozent) }\end{array}$} \\
\hline & & \multicolumn{2}{|c|}{ Wahl SPD-Grüne } & \multicolumn{2}{|c|}{ Wahl CDU-FDP } \\
\hline & & $\begin{array}{c}\text { negative } \\
\text { Bewertung } \\
\text { CDU-BPol. } \\
\text { und positive } \\
\text { Bewertung } \\
\text { SPD-BPol. }\end{array}$ & $\begin{array}{c}\text { positive } \\
\text { Bewertung } \\
\text { CDU-BPol. } \\
\text { und negative } \\
\text { Bewertung } \\
\text { SPD-BPol. }\end{array}$ & $\begin{array}{c}\text { negative } \\
\text { Bewertung } \\
\text { CDU-BPol. } \\
\text { und positive } \\
\text { Bewertung } \\
\text { SPD-BPol. }\end{array}$ & $\begin{array}{c}\text { positive } \\
\text { Bewertung } \\
\text { CDU-BPol. } \\
\text { und negative } \\
\text { Bewertung } \\
\text { SPD-BPol. }\end{array}$ \\
\hline \multirow[t]{3}{*}{1991} & Unabhängige & 64 & 13 & 10 & 51 \\
\hline & Parteianhänger & 93 & 57 & 47 & 86 \\
\hline & $\begin{array}{l}\text { Parteianhänger mit gleicher } \\
\text { Ministerpräsidentenpräferenz }\end{array}$ & 99 & 89 & 71 & 95 \\
\hline \multirow[t]{3}{*}{1999} & Unabhängige & 76 & 15 & 12 & 79 \\
\hline & Parteianhänger & 90 & 53 & 65 & 96 \\
\hline & $\begin{array}{l}\text { Parteianhänger mit gleicher } \\
\text { Ministerpräsidentenpräferenz }\end{array}$ & 96 & 72 & 63 & 97 \\
\hline \multirow[t]{3}{*}{2003} & Unabhängige & 54 & 27 & 27 & 63 \\
\hline & Parteianhänger & 88 & 72 & 50 & 83 \\
\hline & $\begin{array}{l}\text { Parteianhänger mit gleicher } \\
\text { Ministerpräsidentenpräferenz }\end{array}$ & 96 & 91 & 83 & 96 \\
\hline \multicolumn{6}{|c|}{$\begin{array}{l}\text { gleiche Ministerpräsidentenpräferenz = gleichgerichtete Ministerpräsidentenpräferenz; BPol. = Bundes- } \\
\text { politiker. } \\
\text { Eingetragen sind die geschätzten Wahlwahrscheinlichkeiten der Lager für vollkommen negative Bewer- } \\
\text { tungen des CDU-Bundespolitikers und gleichzeitig vollkommen positive Bewertungen des SPD-Bun- } \\
\text { despolitikers sowie für vollkommen positive Bewertungen des CDU-Bundespolitikers und gleichzeitig } \\
\text { vollkommen negative Bewertungen des SPD-Bundespolitikers. } \\
\text { Quelle: Eigene Berechnungen. }\end{array}$} \\
\hline
\end{tabular}


Positive Einstellungen zu Bundespolitikern und zur Bundesregierung können somit einen beachtlichen Einfluss auf das Wählervotum bei Landtagswahlen ausüben, insbesondere in der Gruppe der Unabhängigen, bei der sie zu einer Veränderung der Wahlabsicht führen. Angesichts der in modernen Demokratien zu beobachtenden Abschwächung der Parteiidentifikationen ${ }^{14}$ dürften direkte bundespolitische Einflüsse zukünftig eine noch größere Rolle für das Wählervotum bei Landtagswahlen spielen, weil die Gruppe der Unabhängigen wächst.

\section{Zusammenfassung}

(1) Die Landtagswahlentscheidungen hessischer Bürger wurden hinsichtlich zweier bundespolitischer Einflussfaktoren, nämlich der Zufriedenheit mit der Bundesregierung und der Einstellungen zu Bundespolitikern, sowie der landespolitischen Einflussfaktoren (Zufriedenheit mit der Landesregierung und Einstellung zu Landespolitikern) und der ebenenunspezifischen Parteiidentifikation untersucht.

(2) Sozialdemokratische Parteiidentifikationen sind seit 1991 leicht im Rückgang. Dies ist ein weiteres Indiz für den Niedergang der „roten Bastion Hessen“ ${ }^{15}$. Dagegen steigt der Anteil von Personen ohne Parteiidentifikation beziehungsweise mit einer Parteiidentifikation zu einer Splitterpartei. Die einzelnen Orientierungen sind nicht asymmetrisch zugunsten einer bestimmten Partei verteilt, sondern variieren von Orientierung zu Orientierung und von Wahl zu Wahl. Eine Ausnahme stellt lediglich die Landtagswahl 2003 dar, bei der die CDU auf allen Gebieten vorn lag.

(3) Die erwarteten bivariaten Zusammenhänge zwischen den bundespolitischen Einflüssen und dem Wahlverhalten lassen sich nachweisen: CDU-Bundespolitiker sind bei den Wählern dieser Partei auf Landesebene beliebter als bei SPD-Wählern (und vice versa); setzt sich die Bundesregierung aus den Parteien zusammen, die man im Land gewählt hat, ist man mit der Bundesregierung zufriedener. In einem multivariaten Modell unter Kontrolle der Parteiidentifikation und der landespolitischen Faktoren sind bundespolitische Effekte aber eher zu vernachlässigen.

(4) Bundespolitische Effekte treten im multivariaten Modell vor allem beim Wählertyp der Unabhängigen, also den Personen ohne Parteiidentifikation und Ministerpräsidentenpräferenz, auf. Hier können sie in der Tat wahlentscheidend werden. Vor dem Hintergrund allgemeiner Dealignmentprozesse dürfte damit mit einem Bedeutungsgewinn der bundespolitischen Variablen zu rechnen sein.

14 Siehe Russell J. Dalton, The Decline of Party Identification, in: ders. / Martin P. Wattenberg (Hrsg.), Parties without Partisans. Political Change in Advanced Industrial Democracies, Oxford 2000, S. $19-36$.

15 Gerhard Franz / Robert Danziger / Jürgen Wiegand, a.a.O., S. 64 